\title{
Los desórdenes mentales en el Código Penal chileno: un estudio sobre la inimputabilidad
}

\author{
José Manuel Fernández Ruiz*
}

\begin{abstract}
RESUMEN
El presente trabajo tiene por objetivo enriquecer la comprensión de la imputabilidad, específicamente, conceptualizando los desórdenes mentales, examinando sus clases e identificando las capacidades agenciales que afectan y que son cruciales para poder establecer que una persona es inimputable. Cumplir este objetivo exige reinterpretar el artículo $10 N^{\circ} 1$ del Código Penal chileno. Argumento que este precepto debe ser comprendido de manera que le permita cumplir la función que le corresponde: excluir la culpabilidad cuando el sujeto no posee las capacidades necesarias para una conducta culpable. Propondré una distinción clave entre distintos tipos de desórdenes mentales que le permitirá a dicho precepto cumplir esta función crítica en un derecho penal de la culpabilidad.
\end{abstract}

Desorden mental; inimputabilidad; razonamiento práctico

\section{Mental disorders in the chilean Criminal Code: a study on culpability}

\begin{abstract}
The purpose of this work is to enrich the understanding of culpability, specifically, conceptualizing mental disorders, examining their classes, and identifying the agential capacities that they affect and that are crucial to establishing that a person is culpable. Meeting this goal requires reinterpreting Article $10 N^{\circ} 1$ of the chilean Criminal Code. I argue that this precept must be understood in a way that allows it to perform its proper function: excluding culpability where the subject does not possess the necessary capacities to act culpably. I will propose a key distinction between different types of mental disorders that will allow said precept to fulfil this critical function for a criminal law of culpability.
\end{abstract}

Mental disorder; culpability; practical reason

* Licenciado en Ciencias Jurídicas y Sociales, Universidad de Chile. Magíster en Derecho, Toronto University, Canadá. Doctor en Derecho, Glasgow University, Escocia. Profesor Asistente del Departamento de Derecho Penal, Universidad Alberto Hurtado, e Investigador Asociado VIODEMOS (ANID - Programa Iniciativa Científica Milenio - Instituto Milenio para la Investigación en Violencia y Democracia, VIODEMOS). ORCID: https://orcid.org/0000-0001-7046-3420. Correo electrónico: jmfernandez@uahurtado.cl

Agradezco a los revisores anónimos de la revista por sus observaciones y comentarios. La versión final de este trabajo mejoró sustancialmente gracias a ello.

Artículo recibido el 1.9.2020 y aceptado para su publicación el 14.6.2021. 


\section{INTRODUCCIÓN}

E sta investigación persigue introducir mayor complejidad en la discusión actual acerca de la inimputabilidad, específicamente cuando se basa en un desorden mental. En la literatura jurídico-penal chilena las discusiones pertinentes a la inimputabilidad se han mantenido por largo tiempo en un grado relativo de superficialidad. Esto ha permitido un considerable grado de consenso de las condiciones en donde la inimputabilidad excluye la culpabilidad ${ }^{1}$, pero hay preguntas que no se han formulado y que son necesarias responder para una correcta interpretación de estas condiciones y el modelo que de ellas recepciona el sistema de justicia penal. En efecto, preguntas como, ¿qué es un desorden mental?, ¿qué capacidades afectan los desórdenes mentales?, ¿qué clases de desórdenes mentales regula el Código Penal chileno en atención a las capacidades que estos afectan?, no han sido formuladas por la literatura y sin una respuesta no parece aceptable estimar que el consenso existente se encuentra justificado. Desarrollar algunas respuestas a estas preguntas es el objetivo de la presente investigación. Esta persigue, en primer lugar, conceptualizar qué son los desórdenes mentales. En segundo lugar, especificar las capacidades que estos afectan y cuya ausencia es necesaria para establecer la inimputabilidad. En tercer lugar, elaborar una distinción entre diversos tipos de desórdenes mentales y que se propone es recepcionada por el art. $10 \mathrm{n}^{\circ} 1$ del Código Penal (en adelante CP).

En atención a sus objetivos, la investigación se estructura de la siguiente manera: en la primera sección identifico el tipo de capacidades que característicamente carece quien es inimputable por causa de un desorden mental y, tentativamente, cómo estas son recepcionadas por el CP chileno. En la segunda sección conceptualizo lo que es un desorden mental e identifico los casos de inimputabilidad regulados por el art. $10 \mathrm{n}^{\circ} 1$ del CP, introduciendo la distinción entre caracterizar un desorden mental como condición y como estado. En la tercera sección y final exploro la historia de la locura en general para justificar la afirmación que el CP incorpora a las nociones de estado y condición, para ello seguiré la narrativa desarrollada por la teoría histórica social y crítica.

\section{LA CARENCIA DE “RAZÓN” Y LAS CAPACIDADES PARA LA CONDUCTA IMPUTABLE}

En la doctrina y la jurisprudencia penal chilena hay consenso respecto de cómo se entiende la imputabilidad, concretamente y en una sentencia habitual se la define como "la capacidad de conocer lo injusto y de determinarse según ese conocimiento..."2.

\footnotetext{
${ }^{1}$ Por culpabilidad, entiendo a grandes rasgos la capacidad de la persona para ser un sujeto apropiado de reproche penal, véase SCANLON, 2008. Para este fin, la persona debe tener, al menos, las capacidades que se requieren para poder evitar infringir la prohibición o mandato prescrito por la legislación penal. Para un enfoque afín véase MAÑALCH, 2014.

${ }^{2}$ Bustos, 1994, p. 517; Etcheberry, 1997, p. 278; Politoff, 1997, p. 526; Künsemüller, 2001, p. 180; Garrido, 2003, p. 205; Politoff et al., 2003, p. 294; Cury, 2005, p. 402; Couso, 2006, p. 153; VArgas, 2011, p. 169; NÁquira, 2015, pp. 518-520.
} 
También hay consenso en el efecto de la falta de imputabilidad, a saber, que la consecuencia jurídico penal de una conducta típica y antijurídica de una persona inimputable no puede ser la pena, sino la medida de seguridad cuando el sujeto es considerado, adicionalmente, peligroso de acuerdo con el art. 455 del Código Procesal Penal (en adelante CPP). Es llamativo que la doctrina no haya dicho mucho más sobre aquello en qué consiste la inimputabilidad, específicamente las capacidades que se supone que carece el inimputable y los desórdenes mentales que causan la pérdida o disminución de dichas capacidades ${ }^{3}$. Esto, sobre todo atendiendo a que han pasado casi 150 años desde la incorporación del art. $10 \mathrm{n}^{\circ} 1$, a que el conocimiento científico ha avanzado sustantivamente en el estudio del cerebro, a que la psiquiatría es hoy una disciplina rigurosa, y a que el sistema jurídico y la ciudadanía son muy diferentes a los de aquella época, tanto por razones demográficas, culturales y como por las instituciones chilenas y el diseño de su modelo político y burocrático.

En general, es posible identificar al menos 4 supuestos de inimputabilidad regulados por el CP, dos de estos no serán objeto de esta investigación y que están vinculados a la edad del sujeto activo del delito ${ }^{4}$. En contraste, como veremos con mayor detalle en esta sección, el fundamento de la inimputabilidad de las personas que tienen un desorden mental radica en que no son suficientemente competentes en nuestras interacciones sociales. Las personas inimputables tienen disminuidas las capacidades que los habilitan para ser competentes socialmente y el origen de dicha inhabilidad es el desorden mental, del que dichas personas no son responsables. En otras palabras, las personas inimputables no son responsables de la carencia de las capacidades que los/las habilitan en las interacciones sociales, porque esta es causada por un desorden mental. La dificultad reside en establecer si el art. $10 \mathrm{n}^{\circ} 1$ se refiere efectivamente a estas capacidades, de modo de afirmar que estas rigen (o deben regir) el establecimiento de la inimputabilidad en el proceso penal.

La propuesta es que el art. $10 \mathrm{n}^{\circ} 1$ sí hace referencia a estas capacidades. Para hacer plausible esta afirmación debemos seguir dos pasos. En primer lugar, hay que distinguir dos clases de casos regulados por este precepto: (1) la privación total de razón por causa independiente de la voluntad y (2) la locura o demencia. En segundo lugar, hay que asignar una particular función a los términos "privación de razón" 5 para la interpretación del art. $10 \mathrm{n}^{\circ} 1$. A saber, los términos "privación de razón” permiten interpretar todas las clases de casos (casos tipo (1) y tipo (2)) regulados por el art. $10 \mathrm{n}^{\circ} 1$, porque su función es identificar los tipos de capacidades que el sujeto inimputable carece ${ }^{6}$. Es decir, no es

${ }^{3}$ Una excepción a esto es NÁQUIRA, 2015, pp. 538-548. No obstante, Náquira no profundiza en el tipo de capacidades que los desórdenes mentales afectan, sino que enumera sus características centrales y sintomatología.

${ }^{4}$ Estos son los supuestos de los menores de 14 años (que son absolutamente incapaces de responsabilidad penal) y los mayores de 14 pero menores de 18, y respecto de los/las cuales se estima que aún no han alcanzado el grado de desarrollo cognitivo, volitivo y emocional suficiente para considerarlos/las plenamente responsables.

${ }^{5}$ La controversia sobre si estos términos incluyen o no la actio libera in causa la examino en Autor, 2021.

${ }^{6}$ En un sentido similar, Etcheberry, 1998, p. 280. 
que solo el sujeto "loco o demente" esté privado de razón, sino que cualquiera que sea el tipo de inimputabilidad, para excluir la culpabilidad, esta debe ser una forma total de privación de razón. Veamos entonces con mayor detalle el significado de estos términos.

En principio, la doctrina está relativamente de acuerdo en que no es correcto interpretar "privación de razón" como una privación exclusivamente referida a la carencia de una capacidad cognitiva ${ }^{7}$. La razón por la que esta interpretación sería incorrecta se basaría en la conclusión a la que se llega. Si por "privación de razón" solo puede comprenderse la carencia de una capacidad cognitiva, entonces de ello se seguiría que la incapacidad volitiva, de acuerdo a la cual el sujeto no es capaz de "conformar su comportamiento a su comprensión como uno antijurídico", no calificaría como instancia de inimputabilidad. Si bien el resultado de esta interpretación es correcto, la fundamentación no es completamente satisfactoria. En efecto, no ofrece explicación alguna del porqué las incapacidades volitivas, como por ejemplo las que se manifiestan en la adicción ${ }^{8}$, deben ser comprendidas como una instancia de "privación de razón”. Después de todo, el adicto no tiene, en principio, una incapacidad para conocer que lo que hace esta prohibido. Si el legislador se refiere inequívocamente a la carencia total de "razón”, ¿por qué debemos interpretar el término "razón” como refiriéndose no solo a la carencia de capacidades cognitivas sino, además, de capacidades volitivas?

Una explicación satisfactoria, entonces, debe explicitar en qué sentido la incapacidad volitiva puede considerarse como un caso de "privación de razón". La interpretación que propongo es que "privación de razón" no es equivalente a "incapacidad cognitiva". La incapacidad cognitiva parece estar adecuadamente capturada en la referencia a las capacidades que se requieren para poder afirmar que una persona, digamos $\mathrm{X}$, puede conocer el significado de la antijuridicidad de su conducta. Pero los términos "privación de razón” tienen un significado más amplio, referido al tipo de capacidades que afectan los desórdenes mentales, a saber, aquellas que tradicionalmente nos reconocemos recíprocamente como definitorias de la agencia individual. Más específicamente, la privación que el inimputable experimenta es la capacidad de razonar prácticamente ${ }^{9}$, es decir, la capacidad de establecer "planes" para el futuro ${ }^{10}$. En principio, si esta capacidad se ve comprometida, esto significa que la persona se vuelve incapaz de formar planes para su futuro próximo y distante, satisfacer sus deseos y elegir los medios para satisfacerlos ${ }^{11}$. Esta capacidad para razonar prácticamente es el aspecto determinante que explica por qué el sujeto afectado por un desorden mental es inimputable: ya que no razona correctamente a causa de un desorden mental, no expresa en su conducta las capacidades que

\footnotetext{
${ }^{7}$ Interpretación que la doctrina chilena correctamente rechaza, véase CiLlero, 2011, p. 184.

${ }^{8}$ Acerca de estas incapacidades véase SEbanz y Prinz, 2006.

${ }^{9}$ Moore, 1984, pp. 9-34. Esta capacidad de razonar prácticamente, no obstante, debe comprenderse en un sentido muy amplio, véase la nota a pie $\mathrm{n}^{\circ} 13$.

10 Schopp, 1991, pp. 197-203. Esta concepción de la racionalidad práctica es desarrollada por BRATMAN, 1989.

${ }^{11}$ En general, de este mismo tipo de incapacidades las legislaciones comparadas fundamentan la inimputabilidad, véase SAFFERLING, 2014, pp. 654-677.
} 
se requieren para considerarlo como un agente que se autodetermina y que, por esta razón, puede ser responsable.

Esta interpretación de "pérdida de razón" como equivalente a pérdida de la "capacidad de razonamiento práctico" da cabida, entonces, tanto a incapacidades volitivas como cognitivas. En efecto, la causa de la afectación de la capacidad para razonar prácticamente puede deberse tanto a incapacidades cognitivas (como la incapacidad de saber que lo que se hace está prohibido ${ }^{12}$ ) como volitivas (como la incapacidad de poder controlar los propios deseos), por tanto, ambas incapacidades pueden operar excluyendo o disminuyendo la imputabilidad. De esta manera, podemos identificar con mayor precisión la justificación de los casos que en principio quedan regulados por el art. $10 \mathrm{n}^{\circ} 1$ del CP, a saber, aquellos casos en que el sujeto no es imputable porque tiene un desorden mental que explica la carencia de habilidades cognitivas o volitivas que afectan la capacidad para razonar prácticamente. Cuando esto ocurre, es posible afirmar que no se dan las condiciones para establecer que la persona tiene las competencias suficientes para conducirse en su vida social ${ }^{13}$, en consecuencia, el sujeto no puede ser considerado como un agente característicamente autónomo y responsable. Esta interpretación es consistente con el consenso doctrinario chileno, en el sentido que la imputabilidad incluye dos tipos distintos de capacidades.

Sin embargo, la posición de la doctrina omite especificar lo importante, a saber, las incapacidades que fundamentan la inimputabilidad. La interpretación presente, por el contrario, demuestra el tipo de capacidad general al que responde la diferenciación entre incapacidades volitivas y cognitivas, y explica que es su carencia la que determina la falta de imputabilidad. Como veremos, la interpretación propuesta responde adecuadamente a la categoría de la culpabilidad y se justifica en ella como condición a satisfacer para establecer la responsabilidad penal. Pero, además, demuestra que esta capacidad no solo es algo predicable del caso de aquel que se encuentra privado "totalmente de razón”, sino que se trata de capacidades generales que pueden encontrarse excluidas o disminuidas en cualquier caso de desorden mental y, por tanto, son las mismas capacidades que los términos "locura o demencia" toman como referencia. Podemos concluir al respecto, entonces, afirmando que por "privación de razón” debemos entender la carencia de una

\footnotetext{
${ }^{12}$ Lo característico de la inimputabilidad es que la ausencia de este conocimiento es causada por un desorden mental, véase Schopp, 1991, pp. 33-34.

${ }^{13}$ Hay evidencia empírica que sostiene que ser competente en nuestras prácticas sociales supone ser competente lingüísticamente, porque aquel que maneja correctamente un lenguaje es aquel que demuestra tener un determinado tipo de conocimiento práctico que solo se obtiene tomando parte activa en las prácticas sociales de una determinada comunidad, véase Tomasello, 1999, y Tomasello, 2014. Esta es la razón por la que la capacidad a la que me referí en la sección 1 de este trabajo debe ser entendida en un sentido amplio, al menos más amplio que la comprensión davidsoniana tradicional de la teoría de la acción, que entiende esta capacidad exclusivamente como la habilidad de la persona de guiar su conducta por deseos y creencias proposicionales. Sigo aquí una comprensión más amplia del razonamiento práctico que incorpora la noción wittgenstiana relativa a la capacidad de guiarse y seguir reglas, para la cual esta es una capacidad práctica lingüística no reducible al conocimiento proposicional del lenguaje, véase Coliva, 2018, pp. 337-356.
} 
capacidad irreducible a una capacidad $\operatorname{cognitiva}{ }^{14}$, sino que se refiere tanto a aspectos cognitivos como volitivos, y que se aplica tanto a los casos de "locura o demencia" como a los casos de "privación total de razón por causa independiente de la voluntad".

Esta propuesta para comprender los tipos de casos (1) y (2) como instancias de la carencia de la capacidad de razonamiento práctico permite despejar un problema interpretativo. El problema consiste en la identificación automática del caso tipo (2) con la permanencia del desorden mental y del caso tipo (1) con su transitoriedad. Esta identificación es una tendencia problemática que exhibe la doctrina ${ }^{15}$, y constituye un error importante porque, como veremos en la sección siguiente, ni la permanencia ni la transitoriedad del desorden mental especifican el grado de afectación de las capacidades necesarias para la conducta culpable. Tanto el caso tipo (1) como (2) pueden conducir a la inimputabilidad completa como reducida, porque lo relevante es si la capacidad está afectada de forma "total" o no. Es decir, para excluir completamente la imputabilidad el desorden mental debe ser "total”, pero no en el sentido que hay que constatar la permanencia del desorden mental. El que el desorden sea "total" materializa las condiciones en las que una persona no puede ser considerada culpable, y es total cuando el desorden es lo suficientemente grave como para concluir que el sujeto no es apto para ser socialmente competente porque carece de las habilidades básicas de razonamiento práctico. Al evaluar la inimputabilidad, entonces, debe establecerse que la persona no posee las capacidades requeridas para una conducta culpable, y para ello no es indispensable saber si su condición es permanente o episódica, sino determinar si el desorden mental causa o no una pérdida total de dichas capacidades.

\section{CONCEPTO DE DESORDEN MENTAL Y LA DISTINCIÓN ENTRE ESTADO Y CONDICIÓN}

Una vez identificadas las incapacidades que un sujeto no debe poseer para su caracterización como inimputable, y como se propone que el CP las recepciona, emerge una concepción amplia de las causas de la inimputabilidad reguladas por el art. $10 \mathrm{n}^{\circ} 1 \mathrm{del}$ CP que podemos denominar como "desórdenes mentales". Ahora bien, hay varias formas alternativas de denominación pero, en general, estas presentan problemas que impactan en su plausibilidad y conducen a su rechazo. En primer lugar, deben rechazarse los términos "enfermedad mental". Si el término "enfermedad" alude a una patología entonces

14 Acertadamente NÁquira, 2002, p. 106 y Cillero, 2011, p. 187.

15 Etcheberry, 1997, p. 285 “....si se trata de una privación permanente, estamos en el campo de la locura o demencia...”; GARRIDO, 2003, p, 218 “...es un proceso patológico ...que trae como resultado una intensa alteración de la personalidad del paciente, con cierta permanencia”; CuRY, 2005, p. 423 “...la diferencia entre las enfermedades mentales y la privación total de razón debería encontrarse solo en el carácter más o menos permanente de aquellas...”; VARGAS, 2011, p. 169 “...los locos o dementes suponen privaciones de razón totales, que se distinguen de ese segundo supuesto en la permanencia o no del estado” ; NÁQUIRA, 2015 , p. 535 “...la expresión "loco o demente”, empleada por la ley penal... [implican] verdaderos estados de alienación de carácter permanente”. 
la enfermedad involucra la posesión de una disfuncionalidad biológica. Sin embargo, hay muchos trastornos mentales que no tienen una base en la biología del cerebro. De hecho, la doctrina mayoritaria se inclina aquí por seguir el modelo "mixto" ${ }^{16}$, el que precisamente rechaza comprender los supuestos del art. $10 \mathrm{n}^{\circ} 1$ del CP exclusivamente en términos de la posesión de una patología. Esta es una razón para desechar esta primera acepción. En segundo lugar, también deben rechazarse los términos "enajenación mental", como sugiere Cury $^{17}$. Si bien el CPP utiliza estos términos en los arts. 455 y siguientes, no es correcto que las medidas de seguridad solo se aplican al enajenado mental entendiéndolo como aquel que es "loco o demente" y que tiene un desorden mental permanente. Las medidas de internación, de tratamiento y custodia dependerán de la gravedad del delito, de la peligrosidad y de la seriedad del desorden mental independientemente que este último sea, llegado el caso, transitorio.

Una tercera acepción, recientemente propuesta por Carrasco y Maffioletti ${ }^{18}$, utiliza los términos "trastorno mental". Si bien, en principio, no parece haber diferencias semánticas con los términos "desorden mental" que yo propongo, me parece que en atención al tipo de capacidad que consideran central para efectos de la inimputabilidad, el "juicio crítico", los autores reducen su significado a casos como podrían ser el padecimiento de esquizofrenia, demencia o Alzheimer. En efecto, los autores consideran que lo central para establecer la inimputabilidad por causa de un trastorno mental es la incapacidad de juicio crítico, es decir, aquella capacidad “... del sujeto que le permite distinguir entre el origen intrapsíquico del origen extrapsíquico de sus percepciones, sensaciones o vivencias, distinguir entre el yo y el no-yo, demostrando poder evaluar la realidad de una forma realista" ${ }^{19}$. Sin embargo, estos no son todos los casos regulados por el art. $10 \mathrm{n}^{\circ} 1$. Típicamente ni el psicópata ${ }^{20}$ ni quien tiene un retraso mental severo carece de dicha capacidad de evaluar la realidad de forma "realista", pero un derecho penal de la culpabilidad fundado en las condiciones que hacen apropiado el reproche, exige determinar si estas personas son imputables o no y en qué medida, y no deberían quedar fuera de esta evaluación solamente porque su condición les permite satisfacer en cierta medida el juicio crítico.

En contraste, la noción amplia de "desorden mental" basada en distintas incapacidades de razonamiento práctico puede darles cabida a estos casos, y, por tanto, considerar indispensable su evaluación para determinar la imputabilidad. Ahora bien, todavía no se ha contestado la pregunta acerca de qué es un desorden mental. Para responder a ello sigo la definición que entrega el Manual DSM-5: "Aunque ninguna definición puede capturar todos los aspectos de todos los desórdenes en el rango contenido en el DSM-5, se requieren los siguientes elementos: un desorden mental es un síndrome caracterizado por perturbaciones clínicamente significativas en la cognición, la regulación

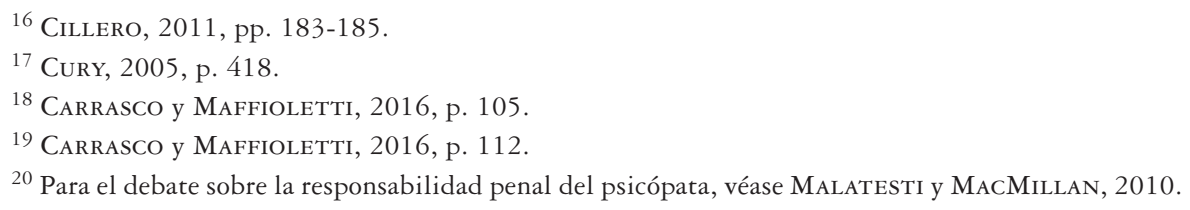


de las emociones o el comportamiento de un individuo que refleja una disfunción en los procesos psicológicos, biológicos o de desarrollo que subyacen al funcionamiento mental. Los desórdenes mentales generalmente se asocian con un malestar o discapacidad importante en actividades sociales, ocupacionales u otras actividades importantes. Una respuesta esperada o aprobada culturalmente a una fuente de estrés o pérdida, como la muerte de un ser querido, no es un desorden mental. Los comportamientos socialmente desviados (por ejemplo, políticos, religiosos o sexuales) y los conflictos que se producen principalmente entre el individuo y la sociedad no son desórdenes mentales, a menos que la desviación o el conflicto sean el resultado de una disfunción en el individuo, como se describió anteriormente ${ }^{21 "}$.

No obstante, la amplitud y cierta vaguedad en la definición de lo que es un desorden mental, la caracterización del DSM-5 para nuestra investigación es suficiente. Especialmente importante es el reconocimiento que el desorden mental no se explica únicamente en una disfunción orgánica del cerebro, sino que hay algún aspecto del ser humano que es apreciado como disfuncional porque “... 2. No funciona de la manera establecida y enseñada de acuerdo a la cultura... 3. No funciona en la forma en que la persona pretende, de acuerdo con sus necesidades y valores, y tal como ella los ve" 22 . Es decir, lo que explica un desorden mental puede estar determinado por el contexto social y cultural. Respecto de esta concepción es posible desarrollar una reinterpretación de las variedades de desórdenes mentales regulados por el art. $10 \mathrm{n}^{\circ} 1$, los casos tradicionalmente comprendidos como tipo (1) y tipo (2). Esta reinterpretación toma como base la distinción entre la noción de estado versus condición mental y que es necesaria para su reconstrucción racional ${ }^{23}$. Básicamente, en el estado, el desorden mental implica que el sujeto, al menos para nuestras prácticas de responsabilidad en sentido amplio y de responsabilidad penal en sentido restringido, es comprendido en general en sus interacciones con otros como una persona no reconocible como un agente en toda su expresión. En la condición, el sujeto a pesar de tener un desorden mental es comprendido en general como un agente.

Podría objetarse que esta propuesta no involucra más que un mero cambio terminológico respecto de los casos tipo (1) y (2), pero estos no son extensionalmente equivalentes a la distinción entre estado y condición porque esta distinción se construye de manera diferente a la idea de permanencia y transitoriedad a la que adhiere la doctrina chilena. Para la literatura chilena, si una persona X es catalogada como un caso tipo (2) debería concluirse que necesariamente tiene un desorden permanente que excluye siempre la imputabilidad. El problema es que, en la medida que se correlacione la permanencia del desorden con la completa ausencia de razonamiento práctico, se dejaría fuera de

21 American Psychiatric Association, 2013, p. 20.

22 Bolton, 2013, p. 443. De manera similar, el manual ICD-11 elaborado por la OMS también reconoce el carácter cultural de ciertos desórdenes mentales, véase GAEBEL et al., 2017, p. 180.

${ }^{23}$ Del método de la "reconstrucción racional”, véase MACCormick, 2005, pp. 29-32. Como precisión, cito este trabajo de MacCormick porque en esta oportunidad se distancia de su previa concepción de reconstrucción racional elaborada por él, contrástese con MACCORMICK et al., 1991, pp. 18-24. 
consideración supuestos que podrían quedar regulados por (2). En efecto, los supuestos del psicópata ${ }^{24}$ y el retraso mental severo ${ }^{25}$, que la literatura penal excluye como casos de inimputabilidad completa, si bien son permanentes no tienen necesariamente el grado de afectación de sus capacidades de razonamiento práctico para considerarlas completamente ausentes. No obstante, el grado de afectación de dichas capacidades sí podría determinar la inimputabilidad del psicópata y la de quien tiene un retraso mental severo. Podría pensarse entonces que estos quedarían regulados por el caso tipo (1), pero esto no sería consistente con la tesis que allí se regulan exclusivamente desórdenes mentales transitorios. Darle cabida a este tipo de supuestos es necesario en un derecho penal de la culpabilidad y ello exige del intérprete una comprensión flexible de los casos tipo (1) y (2). Este es precisamente el propósito de introducir la distinción entre estado ${ }^{26}$ y condición: cambiar el foco de atención desde la transitoriedad/permanencia del desorden a las condiciones por las que la persona debe ser considerada inimputable.

La distinción entre estado y condición no determina por sí misma, a diferencia de la comprensión tradicional de los casos tipo (1) y (2), que el sujeto sea inimputable. En efecto, he afirmado previamente que el criterio para excluir la imputabilidad es que el desorden mental sea "total", es decir, solo si el estado o la condición es total puede excluirse la aplicación de la pena. Si bien es complejo establecer cuándo un desorden mental es total, hay una comprensión general que permite especificar cuando ello ocurre y que es desarrollada por el filósofo PF Strawson en los términos de las perspectivas que denomina participante o reactiva versus la perspectiva terapéutica u objetivante. He afirmado anteriormente que una persona interpretada como agente es aquella que es socialmente competente, lo que desde un punto de vista strawsoniano significa que la persona entiende las prácticas sociales en que toma parte y, en especial, comprende las relaciones sociales que forja y constituye con otros. Strawson pone de relieve que en esta comprensión ocupa un lugar central las intenciones y creencias de los otros/as, y cómo nuestras propias intenciones y creencias dependen de lo que creemos que los otros/as tienen la intención de hacer o decir ${ }^{27}$. Strawson se enfoca en la actitud que expresan las acciones de los otros/as ${ }^{28}$ y cómo las entendemos, porque no es lo mismo si X le pega a $\mathrm{Y}$ en la cara para aplastar una araña peligrosa que estaba muy cerca de picarle, que si $\mathrm{X}$ le pega a $\mathrm{Y}$ sin razón alguna. Nótese, dice Strawson, que el grado de dolor puede

${ }^{24} \mathrm{La}$ literatura penal chilena considera que el psicópata solo tiene disminuida su imputabilidad, véase GARRIDO, 2003, p. 218. Hay que resaltar que esta literatura no considera la gran variedad de casos semejantes al del psicópata, para un examen de estos otros casos véase BARLETT, 2010.

${ }^{25}$ En general la literatura penal chilena solo considera como inimputable al "oligofrénico", ETCHEBERRY, 1997, p. 281; Garrido, 2003, p. 281, Politoff et al., 2003, p. 300; en contra NÁquira, 2015, p. 540.

${ }^{26}$ Nótese que tanto una persona que tiene un retraso mental moderado como alguien que padece Alzheimer tienen un desorden mental caracterizable como estado. A pesar de lo anterior, aquel que tiene un retraso mental moderado todavía es considerado parcialmente como participante, a diferencia de la persona que tiene un estado avanzado de Alzheimer, y por esta razón puede ser considerado como responsable.

${ }^{27}$ STRAWSON, 2008, p. 5.

${ }^{28}$ Strawson, 2008, p. 5. 
ser el mismo en ambos casos, pero en el primer caso Y podría sentirse agradecido, pero no en el segundo.

Este tipo de sentimientos y emociones que se generan como respuestas apropiadas a las acciones realizadas con distintos estados intencionales, constituye lo que Strawson denomina actitudes reactivas. Lo que estas actitudes tienen en común es que hacen que, por ejemplo, en el primer caso, la actitud de Y, de agradecimiento, sea en cierta medida apropiada a la agencia desplegada por X. Uno pudiera preguntar aquí, ¿qué tiene que ver todo esto con los desórdenes mentales? La respuesta es que los casos de desórdenes mentales son precisamente aquellos en que el significado de las actitudes que podemos tener en respuesta a la conducta de $\mathrm{Y}$ son, de alguna manera, inapropiadas. Si Z es quien mata la araña porque $\mathrm{Z}$, bajo una alucinación, le pega a $\mathrm{X}$ en la cara porque pensaba que un fantasma se lo iba a comer, la reacción de $\mathrm{Y}$, tanto de agradecimiento como de enojo, sería inapropiada. Si Y comprende el significado de las razones que llevaron a Z a desplegar su conducta, su actitud reactiva inicial de enojo, ciertamente debería tender a modificarse cuando $\mathrm{Y}$ entiende que dichas razones estaban causadas en un desorden mental ${ }^{29}$.

La concepción de Strawson acerca de la importancia de estas perspectivas facilita entender que, así como la diferencia entre tratar a una persona de forma reactiva versus objetivante es una de grado, el estado y la condición también lo son. En efecto, el estado y la condición son una cuestión de grado y para establecer si disminuyen o excluyen la culpabilidad deberá apreciarse cómo y en qué medida el desorden mental perjudica al sujeto en sus interacciones sociales cotidianas, tal como lo presupone el Manual DSM-5. Esta comprensión del desorden mental que diferencia entre perspectiva participante y perspectiva terapéutica, evita comprender a quien tiene un desorden mental como un simple objeto de técnicas terapéuticas. La comprensión de Strawson enfatiza que, después de todo, se trata de un cambio de perspectivas que puede revertirse cuando quien tiene un desorden ha recuperado sus capacidades agenciales. Asimismo, es consistente con la identificación de casos intermedios como el del psicópata y el retraso mental severo, en que la perspectiva participante y terapéutica se entremezclan. Por último, demuestra consistencia con los manuales DSM-5 y ICD-11 y la doctrina mayoritaria ${ }^{30}$ que de alguna manera rechaza la idea que el desorden mental se pueda reducir a una patología orgánica ${ }^{31}$. Puede haber, en definitiva, desórdenes mentales aun cuando no exista una afectación de la estructura orgánica del cerebro, y así como el Alzheimer, los "pacientes” pueden transitar desde no tener inconvenientes para manejarse de manera competente en su vida cotidiana, a casos en que dicha capacidad está completamente ausente.

Por último, es necesario responder brevemente a otras objeciones. No es una objeción contra la diferenciación propuesta que esta sea imprecisa. En efecto, esto no se sigue de la clasificación sino de la diversidad de los desórdenes mentales que existen.

29 STRAWSON, 2008, p. 9.

${ }^{30}$ Véase NÁQuira, 2015, p. 514-518.

${ }^{31}$ La visión reductivista se aprecia en la influyente definición de enfermedad como fenómeno bioestadístico, véase Boorse, 2011 pp. 13-64. 
Ciertamente hay casos en que es difícilmente categorizable el desorden como estado o condición, pero, además, hay otros en que hay un tránsito temporal desde la condición hacia el estado, como el caso de la demencia. También aparece que esta diferenciación depende en cierta medida de la posibilidad de considerar un desorden como tratable, porque ciertamente una medicina que suprima la sintomatología del Alzheimer, aunque no haga desparecer la condición, si podría hacer desaparecer el estado y, en consecuencia, la persona volver a ser considerada como un participante en nuestras prácticas sociales como agente autónomo y responsable. La presente investigación adopta la interpretación semiconstructivista de la filosofía de la medicina, y no niega su integración con los modernos desarrollos de las ciencias cognitivas. En definitiva, la caracterización de un desorden como estado y condición depende entonces, en parte, del desarrollo de las ciencias cognitivas, terapéuticas ${ }^{32}$ y del propio contexto social y cultural en que la persona se desenvuelve, y es precisamente esta diferencia la que permite reinterpretar de manera más apropiada los casos tipo (1) y casos tipo (2).

\section{TRÁNSITO DEL DESORDEN MENTAL COMO ESTADO A CONDICIÓN EN El Código PENAL}

En esta sección se plantea una explicación histórico-social de la distinción entre un desorden mental caracterizable como estado y como condición en el CP chileno. Esta revela por qué nuestra doctrina y jurisprudencia continúan apegadas a una comprensión del estado como si este fuera inherentemente total, en el sentido que siempre excluye la imputabilidad. Ello se debe, como veremos, a que caracterizar un desorden mental como condición es un desarrollo bastante moderno en las ciencias de la psiquiatría. Esto se revela, tímidamente, en la introducción del art. 10 del CP chileno y en la obra de Pacheco, porque esta exhibe precisamente el problema de identificar el estado con la exclusión de la imputabilidad. Pacheco se esfuerza por interpretar el art. 8 del CP español para diferenciar el caso del "loco o demente", que él entiende que siempre es inimputable, de aquellos casos en que el sujeto solo está privado temporalmente de razón ${ }^{33}$. Es precisamente esta inflexibilidad del art. 8 del CP español la razón por la cual el CP chileno incorpora el caso tipo (1) "Puesto en discusión el art. 10, aceptado en general el principio establecido en el num. $1 .^{\circ}$ del art. $8 .^{\circ}$ del Código español que servía de base, el señor Altamirano opinó porque se diese más latitud a su prescripción comprendiendo otros varios casos análogos como el del sonámbulo” ${ }^{34}$. Es decir, el considerar necesariamente

${ }^{32}$ Este semiconstructivismo es una influyente línea de pensamiento en la filosofía de la psiquiatría y la psiquiatría aplicada, los trabajos de Bolton y Wakefield son paradigmáticos aquí, véase a modo de resumen Bolton, 2014, y WAKefield, 2014.

33 Acertadamente PACHECO, 1881, p. 131-133.

34 Actas de las Sesiones de la Comisión Redactora del Código Penal Chileno, p. 8. 
como inimputable a una persona calificada como "loco o demente" es lo que aparentemente lleva al legislador chileno a incorporar los casos tipo $(1)^{35}$.

Consideremos adicionalmente la noción de intervalos lúcidos en el CP de 1874. Esta noción, abandonada en la ciencia moderna ${ }^{36}$, parecía plausible en un estado incipiente del desarrollo de la psiquiatría. De hecho, la noción de "intervalo lúcido" aparece en el siglo XIX asociada a la situación de mejoría ${ }^{37}$, no a la idea de un tratamiento de la enfermedad mental. Cuando la persona aparentemente mejoraba el desorden mental se consideraba disminuido, y tal situación se calificaba como un intervalo lúcido. Naturalmente, esta forma de entender el desorden mental solo podía tener sentido si se comprendía como un desorden caracterizado como estado ${ }^{38}$. Ya que se trataba del desarrollo incipiente de la medicina en general y la psiquiatría como una de sus especialidades, parecía plausible incorporar la noción de intervalo lúcido. Desde un punto de vista strawsoniano, si el sujeto efectivamente mejoraba su estado, entonces podía entenderse como encontrándose en un intervalo lúcido, y sus interacciones podían interpretarse como la de un participante, y por tanto, aparecía como un agente responsable de sus actos.

Hay un tránsito histórico desde la comprensión del desorden mental como un estado hacia incluir el desorden mental como una condición y que se expresa en la historia institucional chilena. Lo que parece explicar este tránsito es la tardía comprensión de la noción de tratamiento de los desórdenes mentales, como esto se expresa en una determinada forma de las instituciones de salud y por esta razón cómo es recibida desde el punto de vista del sistema jurídico penal. Es decir, hasta hace poco, posiblemente desde después de la década de 1940 en Chile, la concepción del desorden mental no distaba sustantivamente de la comprensión medieval: como una enfermedad incurable. Como estado incurable, el desorden mental sujetaba a los asilos a una comprensión peculiar. Ciertamente, no se puede comprender la institución de los asilos como lugares de tratamiento si se considera el desorden mental como un estado, es decir, como una enfermedad incurable. Vistas las cosas desde un punto de vista strawsoniano, bajo la comprensión medieval, los asilos parecían ser otro lugar más de aquellos donde se abandonaba a quien no se esperaba que pudiera regresar a la perspectiva de participante. En otras palabras, en el siglo XIX, durante el que se introduce el art. 10, la comprensión de tratamiento

${ }^{35}$ Es ciertamente correcto que al introducir el caso tipo (2) el legislador pretendía dar más laxitud interpretativa a la jurisprudencia, pero esto lo hace por el entendimiento previo que las expresiones "loco o demente" se refieren exclusivamente a una situación patológica permanente que elimina completamente la imputabilidad de la persona. Agradezco a uno/a de los/las árbitros la necesidad de aclarar este punto.

${ }^{36}$ Las ciencias cognitivas modernas comprenden los intervalos lúcidos en términos de "fluctuaciones cognitivas" y los estudios empíricos reducen su ocurrencia a ciertos casos de demencia (vascular y Levy) y el Alzheimer, y consideran que, dada su corta duración, de segundos a un par de minutos, y las capacidades que afectan, la capacidad de alerta y atención, y no la memoria, la noción de intervalo lúcido debería abandonarse, véase SHulman et al., 2015, pp. 287-292. En consecuencia, como los términos "intervalo lúcido" no se refieren a ningún tipo de evento no deberían generar efectos jurídico-penales, lo que coincide con la posición de la doctrina mayoritaria, véase Cillero, 2011, p. 191-194, disintiendo Náquira, 2015, p. 529.

37 Berrios, 2012, p. 21.

38 Berrios, 2012, p. 21. 
y cura de la enfermedad mental no había sido completamente desarrollada en los países que lideraban las investigaciones en medicina, y por cierto, no en Chile.

Estos breves datos históricos ilustran preliminarmente algo importante: que la historia del art. 10 se enmarca en una narrativa general de la evolución de la comprensión del desorden mental. Desde luego, no es posible desarrollar con profundidad aquí esta evolución, pero tampoco es necesario. Lo relevante para esta sección es enfatizar que hay un tránsito en la concepción del desorden mental como estado hacia la inclusión de la condición, y que en este tránsito es fundamental la emergencia de la noción de tratamiento o cura. Foucault narra esta evolución con particular lucidez, porque precisamente aborda en su investigación las primeras formas de respuesta social hacia el desorden mental entendido como estado. Foucault básicamente inicia su investigación conectando la respuesta social a la locura con la respuesta social a la lepra, concibiendo ambas como prácticas de exclusión social. Desde su punto de vista, le parece algo más que llamativo que haya sido simultáneo en Europa el desaparecimiento de los leprosarios y el surgimiento de los asilos para las personas con desórdenes mentales, lo que, de acuerdo con Foucault, inicia a fines de la Edad Media. La premisa de Foucault es que categorizar a una persona como alguien que tiene, o sufre, un desorden mental, conlleva una respuesta social que cumple ciertas funciones sociales.

Para Foucault, la respuesta social a la locura, a fines de la Edad Media, estaba asociada originalmente con el pensamiento cristiano del pecado y con el control social de la sinrazón. Sin embargo, con posterioridad, la respuesta social se orienta a la legitimación de la psiquiatría 39 como profesión erigida en "guardián” de los peligrosos, los peligrosos excluidos detrás de las murallas del asilo: "La lepra desapareció, el leproso desapareció, o casi, de la memoria; pero estas estructuras permanecieron. A menudo, en estos mismos lugares, las fórmulas de exclusión serán repetidas, extrañamente similares dos o tres siglos después. Los pobres, vagos, los delincuentes y las 'mentes trastornadas' tomarán el papel del leproso, y veremos qué salvación se esperaba de esta exclusión, para ellos y para aquellos que los excluyeron también. Con un significado totalmente nuevo y en una cultura muy diferente, las formas se mantendrán, esencialmente esa importante forma de una división rigurosa que es la exclusión social y la reintegración espiritual" 40 .

Foucault tiene razón en que la práctica de los asilos, originalmente, porque heredó la función de los leprosarios, era una práctica de exclusión. Sin embargo, esta práctica de exclusión, entendida como la exclusión de la posición de participante ${ }^{41}$, data de mucho antes de la edad media. Ciertamente, durante el imperio Romano la locura se manejaba por la familia ${ }^{42}$, y esta forma de responder a los desórdenes mentales se prolongó, en términos generales, hasta fines de la edad media. Una de las razones aducidas por Foucault que explicaban esta respuesta familiar al manejo del desorden mental, era el

\footnotetext{
39 Foucault, 1990, p. 205.

${ }^{40}$ Foucault, 1990 , p. 7.

${ }^{41}$ No obstante, es posible interpretar que desde el surgimiento de la psiquiatría no solo se han producido exclusiones, sino la inclusión de lo irracional dentro del cuerpo social, véase Jones, 2002, pp. 247-272.

42 Porter, 2003, pp. 89-90; Pietikainen, 2015, p. 82.
} 
estigma social que generaba ${ }^{43}$. La locura, hasta antes del fin de la edad media, permanecía escondida para evitar el estigma que generaba a la familia. La segregación y salida del cuidado familiar comienza a fines de la edad media y es una respuesta generalizada en el sentido que parecía haber un interés público en su regulación. En efecto, hay evidencia histórica de financiamiento por ciertas monarquías del cuidado de la persona con un desorden mental dentro de la familia en Inglaterra ${ }^{44}$ e Italia ${ }^{45}$, así como hay evidencia del tránsito del cuidado fuera de la familia en monasterios y algunas veces financiados centralmente en Europa y Asia durante el siglo XVIII.

La situación, en efecto, cambia radicalmente en Europa durante la Ilustración y el surgimiento incipiente de las ciencias médicas, en particular las primeras nociones de tratamiento desde el siglo XVIII. El asilo, como espacio de control de personas que aparece en esta época y de hecho creó la posibilidad de la emergencia de una nueva disciplina: la psiquiatría. Con el surgimiento de la psiquiatría y la aplicación sistemática del método científico a la explicación de las causas de un desorden mental, se aprecia un desarrollo o tránsito del abandono a la comprensión del asilo como lugar de abandono, a su comprensión como recinto de tratamiento. El tránsito no fue, sin embargo, lineal. En efecto, los historiadores describen el tránsito desde el abandono de la concepción medieval y el inicio de la esperanza del tratamiento con el avance del positivismo y la legitimidad del método científico, hasta el pesimismo ${ }^{46}$ que inicia unas décadas antes del término del siglo XX y que se extiende durante una parte importante del siglo XX. Esta historia tiene, por tanto, retrocesos, idas y venidas. Esto es importante, porque precisamente la noción de tratamiento se inserta en un campo de interacciones sociales en que no siempre quien efectivamente tiene un desorden mental es caracterizado como tal. La historia ha demostrado un frecuente uso arbitrario de la noción de desorden mental, aplicado contra rebeles, revolucionarios, o aquellos considerados inferiores. La psiquiatría y sus formas de tratamiento nunca han estado exentas de polémica.

Finalmente, durante el siglo XX se inicia un nuevo tránsito en el tratamiento del desorden mental, si bien no se abandona el asilo, sí aparecen nuevas formas de tratamiento que tienen por finalidad integrar al sujeto a su comunidad, y que no necesariamente pasan por su internación en un centro especializado. Desde luego, ello también significó un cambio en los tipos de tratamiento de los desórdenes mentales, por ejemplo, comienzan a abandonarse las técnicas de shock eléctrico. El caso de Hemingway es ilustrativo, porque es uno que marca, por lo público del acontecimiento, un cambio en la percepción de los efectos adversos que los tratamientos pueden tener en la vida de los pacientes: "La creciente depresión de Ernst Hemingway (1899-1961) lo llevó a ingresar a la Clínica Mayo en diciembre de 1960, donde recibió una serie de ECT [electroshocks]. Dado de alta

\footnotetext{
43 Foucault, 1990 , p. 67.

${ }^{44}$ Roffe y RofFe, 1995, pp. 1708-1713.

45 Guarnieri, 2009, pp. 34-39.

${ }^{46}$ El tránsito desde la fe en el positivismo hacia el pesimismo varía de país a país, no obstante, en Europa
} este tránsito se sitúa entre mediados del siglo XIX e inicios del siglo XX, véase Plumed y Rojo, 2015, pp. 1-17 en España, Sheperd, 2016, pp. 413-491 en Francia, Harsin, 1992, pp. 1048-1070 en Inglaterra. 
a mediados de enero de 1961, su estado mental siguió siendo frágil hasta su readmisión en abril, cuando nuevamente fue tratado con más terapia de ECT. Dado de alta el 30 de junio, se suicidó dos días después, disparándose con una escopeta en la cabeza. Dejó atrás una denuncia de su tratamiento 'Lo que estos doctores de shock no saben es acerca de los escritores ... y lo que les hacen ... ¿Cuál es el sentido de arruinar mi cabeza y borrar mi memoria, que es mi capital, y dejarme sin trabajo? Fue un tratamiento brillante, pero perdimos al paciente", 47 .

En esta narrativa, aparece que el entendimiento de la locura o demencia como estado tiene lugar en un momento previo al desarrollo de la psiquiatría y su institucionalización dentro de la práctica de la medicina, y previo a la noción de tratamiento. Aparece, asimismo, una particular comprensión del desorden mental en la que se enmarca la concepción del legislador penal chileno tanto del estado como de los intervalos lúcidos, y que tiene su origen en los tiempos previos al positivismo científico, y, por tanto, previos a la parte final de la Ilustración. Es la época previa, propia del término de la Edad Media en que todavía se comprende el desorden mental como estado y en el que la respuesta social pareciera encaminarse primordialmente a la comprensión de los asilos como lugares donde las familias pueden controlar el estigma social. Respalda esta narrativa histórica el grado de desarrollo de las ciencias médicas y psiquiátricas en Chile a la época, y el surgimiento de sus primeros asilos. En efecto, en Chile el primer recinto para enfermos mentales se fundó en 1852, aunque la profesionalización de la medicina como carrera inicia unos años antes, en $1833^{48}$. Más tardíamente aparece la carrera de psiquiatría ya institucionalizada como curso impartido por la Universidad de Chile, donde se inaugura en $1927^{49}$. Este retraso en la profesionalización de la psiquiatría sugiere que la creación del recinto en 1852 se sitúa, a nivel discursivo, de manera similar a los recintos para desórdenes mentales de Europa, a saber, bajo la ideología medieval. En otras palabras, sin un cuerpo de profesionales con estudios relativamente profundos acerca de la psiquiatría y la enfermedad mental, los recintos en Chile parecen haber continuado bajo el alero de dicha ideología ${ }^{50}$. Esto tiene sentido, porque la noción de tratamiento surge con la emergencia de las ciencias médicas en Europa y su desarrollo, lo que tiene lugar en una fecha posterior.

Como han evidenciado estudios de historia social de la psiquiatría ${ }^{51}$, es necesaria la emergencia de cuerpos profesionales para la formación de instituciones propiamente

${ }^{47}$ SCULL, 2015, p. 318.

48 Araya, 2018, p. 149.

49 Araya, 2018, p. 150. Pero el surgimiento de la carrera tampoco demuestra que a la fecha ya existía un número considerable de profesionales y un cuerpo médico profesionalizado que efectivamente posibilitara el tratamiento de los pacientes.

${ }^{50}$ La noción de tratamiento con miras a la cura, como afirma Scull, es un aspecto importante de la legitimación de la psiquiatría como disciplina, SCULL, 2006, pp. 129-130. En general, para el rol del control del conocimiento en los sistemas profesionales véase Аввотт, 1988.

${ }^{51}$ El hospital posiblemente más influyente en la historia europea de la psiquiatría fue el Salpetriere en Francia, donde Philipe Pinel y sus discípulos le dieron forma al tratamiento psiquiátrico de los enfermos mentales (Micale, 1985). Esta "historia institucional" es importante porque hay evidencia en Chile que en 
tales, como las instituciones de salud y por tanto las nociones de tratamiento. La historia europea de la que se asienta la estructura del Estado-Nación, modelo de organización política importado a Chile por los independentistas, y la evidencia histórica acerca de la evolución de las ciencias médicas en Chile a la psiquiatría, sugieren que el contexto en el que el art. $10 \mathrm{n}^{\circ} 1$ tiene su origen, en 1873, estaba dominado por la ideología medieval, en que el desorden mental era un estigma social, en que una vez surgido los asilos los enfermos eran abandonados, y que por tanto, no existiendo cura, su situación era entendida casi exclusivamente como estado. Como hemos visto, es ilustrativo de esta comprensión el texto de Pacheco, quien de hecho comprende los términos loco o demente como "...la absoluta y de ordinario constante falta de la razón, ese primer elemento de la humanidad, ese primer sello del hombre" 52 .

Finalmente aparecen las nociones de curabilidad y tratamiento en la historia de las instituciones chilenas, con la emergencia del positivismo científico y la legitimidad del método científico como forma de diagnosticar y diseñar tratamientos para los desórdenes mentales ${ }^{53}$. El inicio y posterior consolidación de las ciencias médicas y psiquiátricas marca, en consecuencia, un tránsito en la comprensión del desorden mental como estado a incluir la comprensión como condición. Si bien no es la misma comprensión del legislador chileno al incorporar (2), porque este alude inicialmente a los episodios transitorios de pérdida de razón, este tránsito permite reinterpretar el art. 10, porque entender el desorden mental como condición significa comprender que este afecta solo cierta parte de la vida mental de las personas, y ello facilita entender que lo mismo podría ocurrir en los casos de estado no totales. De esta manera, es posible interpretar el art. 10 en conformidad con un derecho penal de la culpabilidad. Para este derecho las personas que tienen un desorden mental deben ser reconocidos como participantes en nuestras prácticas sociales, y, por tanto, en general, siempre queda abierta la consideración de sus acciones en términos de actitudes reactivas. En otras palabras, no siempre es posible negar la responsabilidad penal por sus conductas constitutivas de delito.

el siglo XIX, especialmente en materia civil, existía la práctica de pericias médicas que facilitaban la decisión jurisdiccional en procesos de interdicción por demencia, véase Correa, 2013, pp. 571-585. Sin embargo, la experiencia comparada demuestra que no basta una práctica como la descrita por Correa (lo que ella desde luego no afirma) para poder afirmar la existencia de la psiquiatría como profesión en el siglo XIX, y menos que el paradigma ideológico bajo cual dicha práctica tenía lugar era positivista. En Salpetriere, como lo demuestra Micale, ello recién tiene lugar a fines del siglo XIX. En Chile, a la época, todavía el único hospital de orates luchaba contra la escasez de médicos y las condiciones mínimas de habitación para los pacientes, para un examen de las condiciones sanitarias de estos recintos véase CAMUs, 1993, pp. 136-137. El proceso de institucionalización que en Salpetriere inicia a fines del siglo XIX, en Chile es posterior a 1930. En efecto, el proceso de institucionalización no solo de la psiquiatría, sino del primer establecimiento para el tratamiento de personas con enfermedades mentales, se inicia desde 1891 a 1931, pero el proceso de consolidación de dicha institucionalización es posterior a la creación de la cátedra de psiquiatría de la Universidad de Chile en 1927, véase Escobar, 2002. Esto sugiere que la evolución de los procesos de institucionalización de la psiquiatría son simultáneamente locales y globales, véase Alarcón, 2012, p. 516.

\footnotetext{
52 Pacheco, 1881, p. 135.

53 SCUll, 2015, pp. 206-207.
} 


\section{CONCLUSIÓN}

En este trabajo he identificado las capacidades que característicamente afectan los desórdenes mentales, he conceptualizado dichos desórdenes y como resultado ha emergido una reinterpretación más compleja de los casos regulados por el art. $10 \mathrm{n}^{\circ} 1$ del $\mathrm{CP}$ chileno respetuosa del principio de culpabilidad. En efecto, he sostenido que es una exigencia del principio de culpabilidad que la persona sea considerada como participante porque es en tanto participante lo que hace a la persona un sujeto apropiado del reproche penal. Esto implica que lo que afecta el desorden mental y que es relevante para la responsabilidad penal son aquellas capacidades de razonamiento práctico y que son necesarias para una conducta socialmente competente. He argumentado que los términos "privación de razón” precisamente capturan esta comprensión, y que el término "total" hace referencia al grado en que dichas capacidades deben estar afectadas para considerar que la persona es inimputable. He despejado, con ello, el problema de identificar los casos tipo (2) con supuestos de permanencia que siempre excluyen completamente las capacidades de razonamiento práctico.

He elaborado, por último, una interpretación del art. 10 del CP que es capaz de integrarse con los nuevos conocimientos científicos disponibles en materia de salud mental, y para ello ha sido fundamental narrar con cierto detalle la historia general de la evolución de la psiquiatría en Chile. Esta historia social me ha permitido explicar la comprensión de ciertos términos utilizados por el legislador de la época, de modo de poder justificar el tránsito de la comprensión del desorden mental como un estado hasta incluir su comprensión como una condición, y cómo esta distinción es capturada por la regulación del CP. Este tránsito debería, además, tener implicancias para repensar en general las medidas de seguridad. El tránsito histórico del tratamiento social del desorden mental marca también un tránsito en la historia de la consideración de la persona, desde su consideración como un objeto de estigma familiar y exclusión, a una persona cuya agencia debe ser respetada incluso durante el tratamiento. El reconocimiento de la agencia de la persona pone entredicho la comprensión de la medida de seguridad concebida exclusivamente en términos de control de un peligro, porque en la gran mayoría de los casos, el sujeto todavía debería ser considerado como participante.

\section{BIBLIOGRAFÍA}

Аввотt, Andrew, 1998: The System of Professions, Chicago, University of Chicago Press.

Alarcón, Renato, 2012: "Contexto histórico y cultural del estudio de la enfermedad mental: perspectivas latinoamericanas", Revista de Medicina Clínica las Condes, Vol. 23, No 5.

Actas de las Sesiones de la Comisión Redactora del Código Penal Chileno, 1873, Santiago, Imprenta de la República.

American Psychiatric Association, 2013: Diagnostic and statistical manual of mental disorders, (Fifth Edition) Washington, American Psychiatric Publishing.

Araya, Claudia Ibacache, 2018: "Aspectos de la profesionalización de la psiquiatría en Chile, siglos XIX y XX”, Autoctonía. Revista de Ciencias Sociales e Historia, Vol. 2, No 1. 
Barlett, Peter, 2010: "Stabbing in the dark: English law relating to psychopathy", en Malatesti, L y McMillan, J, Responsibility and Psychopathy: Interfacing Law, Psychiatry and Philosophy, Oxford, Oxford University Press, pp. 25-40.

Berrios, German, 2012: "Formation and meaning of mental symptoms: history and epistemology", Dialogues in Philosophy, Mental and Neuroscience, Vol. 6, No 2.

Bolton, Derek, 2013: "What is mental illness", en Fulford, E, Davies, K, Gipps, M, Graham, R y Sadler, G, The Oxford Handbook of Philosophy and Psychiatry, Oxford, Oxford University Press, pp. 434-450.

BolTon, Derek, 2014: "Should mental disorders be regarded as brain disorders? 21st century mental health sciences and implications for research and training", World Psychiatry, Vol. 12, $\mathrm{N}^{\circ} 1$.

Boorse, Christopher, 2011: "Concepts of health and disease", en Gifford, F, Philosophy of medicine, Amsterdam y Oxford, Elsevier, pp. 13-64.

Bratman, Michael, 1989: Intention, plans, and practical reason, Stanford, CSLI Publications.

Bustos, Juan, 1994: Manual de derecho penal parte general, Barcelona, PPU.

Camus, Pablo, 1993: "Filantropía, medicina y locura: la casa de orates de Santiago 1852-1894", Historia, Vol. 27.

Carrasco Jiménez, Edison y Maffioletti, Francisco Celedón, 2016: "Problemas conceptuales y terminológicos en el tratamiento del 'trastorno mental' por el artículo 10.1 del Código Penal chileno", Actualidad Penal, Vol. 244.

Cillero, Miguel, 2011: “Art. 10 n 1”, en Cillero, M, Couso, J, Hernández, H y Mera, J, Código penal comentado, Libro I, Santiago, LegalPublishing Chile, pp. 177-201.

Colrva, Analissa, 2018: "Practical Knowledge and Linguistic Competence", en Coliva, A y Moruzzi, S, Eva Picardi on Language, Analysis and History, London, Palgrave Macmillan, pp. 337-356.

Correa Gómez, María José, 2013: "De la Casa de Orates al juzgado: pericia alienista y evaluación judicial de la locura en Santiago de Chile hacia 1860", História, Ciências, Saúde - Manguinhos, Vol. $20 \mathrm{~N}^{\circ} 2$.

Couso, Jaime, 2006: Fundamentos del derecho penal de culpabilidad, Valencia, Tirant Lo Blanch.

Cury, Enrique, 2005: Derecho penal parte general, (Séptima Edición), Santiago, Editorial Jurídica de Chile.

Escobar, Enrique, "Historia del Hospital Psiquiátrico (1852-1952)". Disponible en http:// www.bibliotecaminsal.cl/wp/wp-content/uploads/2013/08/HISTORIA-HOSPITALPSIQUIATRICO.pdf. [Fecha de consulta: 28 de agosto de 2020].

Etcheberry, Alfredo, 1997: Derecho penal parte general, (Tercera edición), Tomo I, Santiago, Editorial Jurídica de Chile.

Autor, 2021: "Criterios de evaluación para los modelos del tipo y de la excepción en la actio libera in causa", Opinión Jurídica, Vol. 20 No 41.

Foucault, Michel, 1965: Madness and civilization. A history of insanity in the age of reason, New York, Random House.

Gaebel, Wolfgang, Zielasek, Jürgen y Reed, Geoffrey M., 2017: "Mental and Behavioural Disorders in the ICD-11: Concepts, Methodologies, and Current Status", Psychiatria Polska, Vol. 51, N².

Garrido Montt, Mario, 2003: Derecho penal parte general, (Tercera edición), Tomo II, Santiago, Editorial Jurídica de Chile.

Guarnieri, Patrizia, 2009: "Per una storia dell'affidamento dei malati psichiatrici alle famiglie", Epidemiology and Psychiatric Sciences, Vol. 18, Nº 1.

Harsin, Jill, 1992: "Gender, Class, and Madness in Nineteenth-Century France”, French Historical Studies, Vol. 17, $\mathrm{N}^{\circ} 4$. 
Hernández, Héctor, 2011: “Art. 1”, en Cillero, Miguel, Couso, Jaime, Hernández, Héctor, Mera, Jorge, Código penal comentado, Libro I, Santiago, LegalPublishing Chile, pp. 7-123.

Jones, David, 2002: "Madness, the family and psychiatry", Critical Social Policy, Vol. 22, No 2.

Joshi, Jubert, 1989: "Algunas consecuencias que la inimputabilidad puede tener para la antijuridicidad”, Anuario de derecho penal y ciencias penales, Vol. 42, No 1 .

KüNSEMÜLler, Carlos, 2001: Culpabilidad y pena, Santiago, Editorial Jurídica de Chile.

MacCormick, Neil, 2005: Rhetoric and Rule of Law. A Theory of Legal Reasoning, Oxford, Oxford University Press.

MacCormick, Neil, Bankowski, Zenon, Summers, Robert y Wroblewski, Jerzy, 1991: "On Method and Methodology", en Interpreting Statutes: A Comparative Study, Brookfield, Darmouth Publishing.

Malatesti, Luca y McMillan, John, 2010: Responsibility and Psychopathy: Interfacing Law, Psychiatry and Philosophy, Oxford, Oxford University Press.

MaÑalich Raffo, Juan Pablo, 2014: “Las dimensiones de la culpabilidad jurídico-penal”, en Estudios Sobre la Fundamentación y la Determinación de la Pena, Santiago, Thomson Reuters, pp. 5-24.

Micale, Mark, 1985: "Salpetriere in the Age of Charcot- An institutional Perspective on Medical History in the Late Nineteenth Century", Journal of Contemporary History, Vol. 20, No 4.

Moore, Michael, 1984: Law and Psychiatry. Rethinking the relationship, Cambridge, Cambridge University Press.

NÁquira, Jaime, 2015: Derecho penal. Parte general, Santiago, Thomson Reuters.

Náquira, Jaime, 2002: “Artículo 10 n 1”, en Bustos, J, Politoff, S, Ortiz, L y Matus, Jean, Texto y comentario del Código Penal chileno, Santiago, Editorial Jurídica de Chile, pp. 101-107.

Pacheco, Fransisco, 1881: El Código Penal, Concordado y Comentado, (Quinta Edición), Tomo I, Madrid, Imprenta y Fundición de Manuel Tello.

Pietikainen, Petteri, 2015: Madness. A History, London, Routlegde.

Plumed Domingo, José Javier y Rojo Moreno, Luis Miguel, 2016: "The treatment of madness in the nineteenth and twentieth centuries- discourses about curability in Spanish mental health care, 1890-1917", História, Ciências, Saúde - Manguinhos, Vol. 23, № 4.

Politoff, Sergio, Matus, Jean Pierre y Ramírez, María Cecilia, 2003: Lecciones de derecho penal chileno, Santiago, Editorial Jurídica de Chile.

Politoff, Sergio, 1997: Derecho Penal, Tomo I, Santiago, Editorial Conosur.

Porter, Roy, 2003: Madness. A brief history, Oxford, Oxford University Press.

Roffe, David y Roffe, Christine, 1995: "Madness and care in the community: a medieval perspectives", British Medical Journal, Vol. 311.

Sebanz, Natalie y Prinz, Wolfang, 2006: Disorders of Volition, Cambridge Mass., MIT Press.

SAFferling, Christoph, 2014: "Insanity and Intoxication", en Dubber, M y Hornle, T, The Oxford Handbook of Criminal Law, Oxford, Oxford University Press, pp. 654-677.

Scanlon, TM, 2008: Moral Dimensions. Permissibility, Meaning, Blame, Cambridge Mass., Harvard University Press.

ScHopp, Robert, 1991: Automatism, Insanity, and the Psychology of Criminal Responsibility, Cambridge, Cambridge University Press.

SCUll, Andrew, 2006: "Psychiatric therapeutics and the historian", En, The Insanity of Place, the Place of Insanity, London, Routledge, pp. 129-149.

Scull, Andrew, 2015: Madness in civilization, Princeton, Princeton University Press.

ShePerd, Jade, 2016: "I am very glad and cheered when I hear the flute': The Treatment of Criminal Lunatics in Late Victorian Broadmoor", Medical History, Vol. 60, No 4.

Shulman, Kenneth, Hull, Ian, DeKoven, Sam, Amodeo, Sean, Mainland, Brian y Herrmann, Nathan, 2013: "Cognitive Fluctuations and the Lucid Interval in Dementia- Implications 
for Testamentary Capacity", The Journal of the American Academy of Psychiatry and the Law, Vol. 43, $\mathrm{N}^{\mathrm{o}} 3$.

STrawson, PF., 2008: Freedom and resentment and other essays, London, Routledge.

Tomasello, Michael, 1999: Cultural Origins of Human Cognition, Cambridge Mass., Harvard University Press.

Tomasello, Michael, 2014: Natural History of Human Thinking, Cambridge Mass., Harvard University Press.

Vargas, Tatiana, 2011: Manual de Derecho Penal Práctico. Teoría del Delito con Casos, Santiago, LegalPublishing.

WAKEFIELD, Jerome, 2014: "Wittgenstein's nightmare, why the RDoC grid needs a conceptual dimension”, World Psychiatry, Vol. 13, N 1 . 